\title{
Nucleocytoplasmic shuttling of HIV-1 integrase is controlled by the viral Rev protein
}

\author{
Aviad Levin, ${ }^{2}$ Zvi Hayouka, ${ }^{2}$ Assaf Friedler ${ }^{2}$ and Abraham Loyter ${ }^{1, *}$ \\ 'Department of Biological Chemistry; The Alexander Silberman Institute of Life Sciences; and Institute of Chemistry; The Hebrew University of Jerusalem; Jerusalem, Israel
}

Key words: rev, integrase, nuclear import, nuclear export, HIV-1, peptides

\begin{abstract}
In the current study we show that the Rev protein of Human Immunodeficiency Virus type 1 (HIV-1) inhibits nuclear import and mediates nuclear export of the HIV-1 integrase (IN) protein, which catalyzes integration of the viral cDNA. Interaction between IN and Rev in virus infected cells, resulting in the formation of a Rev-IN complex, has been previously described by us. Here we show that nuclear import of the IN, is inhibited by early expressed Rev. No nuclear import of IN was observed when Rev-overexpressing cells were infected by wild-type HIV-1. Similarly, no translocation of IN into nuclei was observed in the presence of Rev-derived peptides. On the other hand, massive nuclear import was observed following infection by a $\Delta$ Rev virus or in the presence of peptides that promote dissociation of the Rev-IN complex. Our results show that IN is only transiently present within the nuclei of infected cells. Treatment of infected cells with leptomycin B caused nuclear retention of the Rev-IN complex. Removal of the leptomycin from these treated cells resulted in nuclear export of both Rev and IN. On the other hand, disruption of the nuclear located Rev-IN complex resulted in export of only the Rev protein indicating Rev-mediated nuclear export of IN. Our results suggest the involvement of Rev in regulating the integration process by limiting the number of integration events per cell despite the presence of numerous copies of viral cDNA.
\end{abstract}

\section{Introduction}

Following fusion of the Human Immunodeficiency Virus type 1 (HIV-1) envelope with the recipient cell plasma membrane, the two viral RNA molecules are reversed-transcribed by the viral reverse transcriptase (RT). This results in the formation of numerous unintegrated viral cDNA molecules. ${ }^{1,2}$ Integration of the cDNA molecules into the host chromosomal DNA is mediated by the viral enzyme integrase (IN), ${ }^{3}$ which is packed within the invading virus particles and released into the infected host cells. ${ }^{4,5}$ HIV-1 IN catalyzes the integration reaction via two successive steps: the 3'-end-processing step, in which a dinucleotide is removed from the viral long terminal repeat (LTR) within the cytoplasm, ${ }^{6,7}$ and a strand-transfer step in which the viral DNA is inserted into the host chromosomal DNA.,8-10 The strandtransfer step necessitates nuclear import of viral IN-DNA complex or of the complete pre-integration complex (PIC). The exact molecular mechanism as well as the viral and cellular proteins required for nuclear import of these complexes are still unclear and controversial. ${ }^{11-19}$

Several studies have shown that despite the fact that each infected cell contains numerous copies of both IN and viral cDNA molecules, only a limited number (1 or 2 ) integration events can be detected per cell. ${ }^{1}$ Recently, we suggested that the limited number of integration events observed in HIV-1infected cells is due to Rev-induced inhibition of IN enzymatic activity. ${ }^{20,21}$ The HIV-1 Rev is a 116 residues protein, which has been assumed to be expressed after the integration process is complete. ${ }^{22}$ It bears a well-defined nuclear localization signal (NLS) and nuclear export signal (NES) sequences that enable its nucleocytoplasmic shuttling. ${ }^{22} \mathrm{Rev}$ has been implicated in nuclear export of unspliced and partially spliced viral RNA molecules following its specific interaction with a Rev-responsive element within the viral transcripts. ${ }^{22}$ Thus it appears that the viral IN and Rev operate at two completely different stages of the viral life cycle. ${ }^{3,22,23}$ However, there is strong accumulating evidence that Rev transcripts can also be expressed from unintegrated viral cDNA, resulting in the appearance of Rev molecules before the initiation of integration. ${ }^{20,24-26}$ For example, Rev can be easily detected in cells infected by a mutant HIV-1 bearing an inactive IN, confirming expression from unintegrated cDNA molecules. ${ }^{20,24-26}$

We have previously shown that the HIV-1 Rev and IN proteins interact with each other and form Rev-IN complexes in virus-infected cells ${ }^{20,27}$ as well as in vitro. ${ }^{28}$ The in vitro experiments revealed that the interaction with Rev inhibits IN enzymatic activity. ${ }^{21}$ Rev-derived peptides (Rev 13-23 and Rev 53-67) also inhibited the enzymatic activity of the HIV-1 IN. ${ }^{28}$ These cell-permeable Rev-derived peptides inhibited integration of the viral cDNA in virus-infected cells. ${ }^{20,21,28}$ Inhibition of viral DNA integration was also observed when cells overexpressing Rev were infected with the wild-type (WT) HIV, suggesting an 
intracellular Rev-IN interaction. ${ }^{20}$ The notion that formation of the Rev-IN complex induces inhibition of IN in virus-infected cells can also be inferred from experiments showing that disruption of these complexes results in stimulation of integration..$^{20,21}$ This may be achieved by IN-derived peptides (INrs), which promote dissociation of the Rev-IN complexes in vitro ${ }^{21}$ as well as in vivo. ${ }^{20}$ Support for the view that the limited number of integration events in HIV-infected cells is due to the presence of an inhibitory Rev was also obtained from experiments showing multiple integration of cDNA following infection by a $\Delta$ Rev virus. ${ }^{20}$

In the present work, we used immunostaining and western blot analysis of isolated cellular fractions to study the intracellular localization and fate of IN, Rev and the Rev-IN complexes in virus-infected cells. We show that right after infection, IN and Rev are restricted to the cytoplasm. At later times, of about $10 \mathrm{~h}$ post-infection (PI), some of the IN and Rev molecules are found within the nuclei of the infected cells. At 16 to $20 \mathrm{~h}$ PI IN is found exclusively in the cytoplasm, in contrast to Rev that is found throughout the cell environment. No intranuclear IN was found following infection of Rev-overexpressing cells, indicating Rev-induced inhibition of IN nuclear import. On the other hand, IN was permanently accumulated within the nuclei following infection with the $\Delta \mathrm{Rev}$ virus. Accumulation of intranuclear IN was also observed in cells infected with the Rev M10 virus, whose Rev NES sequence is mutated ${ }^{29}$ or following treatment of WT-infected cells with leptomycin B, which inhibits Rev Nuclear Export. ${ }^{30}$ In summary, our present results demonstrate the involvement of Rev in inhibiting IN nuclear import and mediating IN nuclear export.

\section{Results}

HIV-1 IN is transiently present within the nuclei of infected cells. Immunofluorescence staining of the IN and Rev proteins in HIV-1-infected HeLa-P4 cells revealed co-localization of these two proteins, indicating the formation of an intracellular Rev-IN complex (Fig. 1A and reviewed in ref. 20). Both the IN and low levels of the expressed Rev were present within the cytoplasm at $6 \mathrm{~h}$ PI. In contrast, most of the cellular Lens Epithelium Derived Growth Factor p75 (LEDGF/p75) protein was localized within the nuclei of the infected cells. Monitoring the localization of IN at different times PI revealed that it became intranuclear only at $10 \mathrm{~h} \mathrm{PI}$, while at 16 and $20 \mathrm{~h}$ PI it was exclusively cytoplasmic, indicating either nuclear export or degradation (or both). A somewhat different picture was obtained when the localization of Rev and LEDGF/p75 was studied (Fig. 1A). Between 10 and $20 \mathrm{~h}$ PI, Rev was present throughout the entire cell while LEDGF/ p75 was localized mainly to the nuclei. We have shown before that the ability of IN to integrate the viral cDNA is regulated by interplay between the inhibitory viral Rev and the stimulatory LEDGF/p75 proteins. $^{27}$

Western blot analysis confirmed the results obtained by the immunofluorescence staining regarding the intracellular localization of IN and Rev, as well as of the intranuclear localization of the cellular LEDGF/p75 (Fig. 1B and for quantitative analysis see Tables S1 and S2). An increase in the amount of the IN was observed at increasing time of infection in all experiments. This may be due to the fact that infection is not synchronized and may last for a relatively long time, especially in case when the added virus is not removed after adsorption. Essentially the same cytoplasmic and nuclear localization patterns were obtained for IN and Rev in infected LEDGF/p75-knockdown cells (Fig. 1C and D), eliminating the possibility that LEDGF/p75 participates in the nuclear import of IN.

The interaction between Rev and IN inhibits nuclear import of IN. When a $\Delta$ Rev virus was used to infect the HeLa-P4 cells, an entirely different picture emerged (Fig. 2A). Already at $6 \mathrm{~h} \mathrm{PI,}$ the viral IN could be seen within the nuclei of the infected cells. Even at $20 \mathrm{~h}$ PI, IN continued to accumulate within the nuclei and appeared to be intranuclear. Essentially the same results were obtained when the intracellular localization of IN was monitored by western blot analysis (Fig. 2B and for quantitative analysis see Tables S1 and S2). When Rev-overexpressing cells were infected with the WT HIV, no nuclear accumulation of IN was observed by either immunofluorescence staining (Fig. 2C) or western blot analysis (Fig. 2D), indicating Rev-induced inhibition of IN nuclear import. In contrast to IN, the Rev protein was detected throughout the cell (Fig. 2C and D).

Inhibition of IN nuclear import by the Rev protein was confirmed by co-expression of both proteins in transfected cultured HeLa cells (Fig. 2E). In cells expressing only IN or Rev, the majority of the expressed proteins were localized in the nuclei. On the other hand, when both proteins were co-expressed, no nuclear import was observed and both proteins appeared to be localized within the cytoplasm (Fig. 2E).

When the intracellular localization of the viral DNA was quantitatively estimated (Fig. 3), a similar pattern to that described in Figure $2 \mathrm{~A}-\mathrm{D}$ was observed. In cells infected by the WT virus, about $20 \%$ of the viral DNA was intranuclear at $16 \mathrm{~h} \mathrm{PI} \mathrm{(com-}$ pare results in Fig. 3A and B). On the other hand, similar to the IN protein (Fig. 2), in cells infected with the $\Delta$ Rev virus, about $80 \%$ of the viral DNA was found within the nuclei of the infected cells. In Rev-overexpressing infected cells, almost no viral DNA was intranuclear, meaning that most of it remained cytoplasmic (Fig. 3A and B). Estimation of viral 2LTR circles (which are present only in the nuclei of infected cells $s^{5}$ confirmed that in cells infected with the $\Delta \operatorname{Rev}$ virus, the quantity of these molecules was much higher than in WT-infected cells. No 2LTR circles were detected in Rev-overexpressing-infected cells (Fig. 3C).

Similar to the Rev protein (Fig. $2 \mathrm{C}$ and D), the two previously described $^{28}$ IN-interacting Rev-derived peptides [Rev 13-23 (Fig. 4A and B) and Rev 53-67 (not shown)], blocked nuclear import of IN without affecting the intracellular localization of Rev. The opposite effect, inhibition of Rev and augmentation of IN nuclear import, was observed following the addition of the Rev-interacting IN-derived peptides ${ }^{21} \mathrm{INr}-1$ (Fig. 4C and D) and INr-2 (not shown). The INrs are cell-permeable peptides that promote dissociation of the intracellular Rev-IN complex, ${ }^{20,21}$ thus probably allowing nuclear import of the released IN. No intranuclear IN was observed in INrs-treated cells at $20 \mathrm{~h}$ PI. This may be because the peptides may be proteolytically degraded by then and thus their effect is lost. 


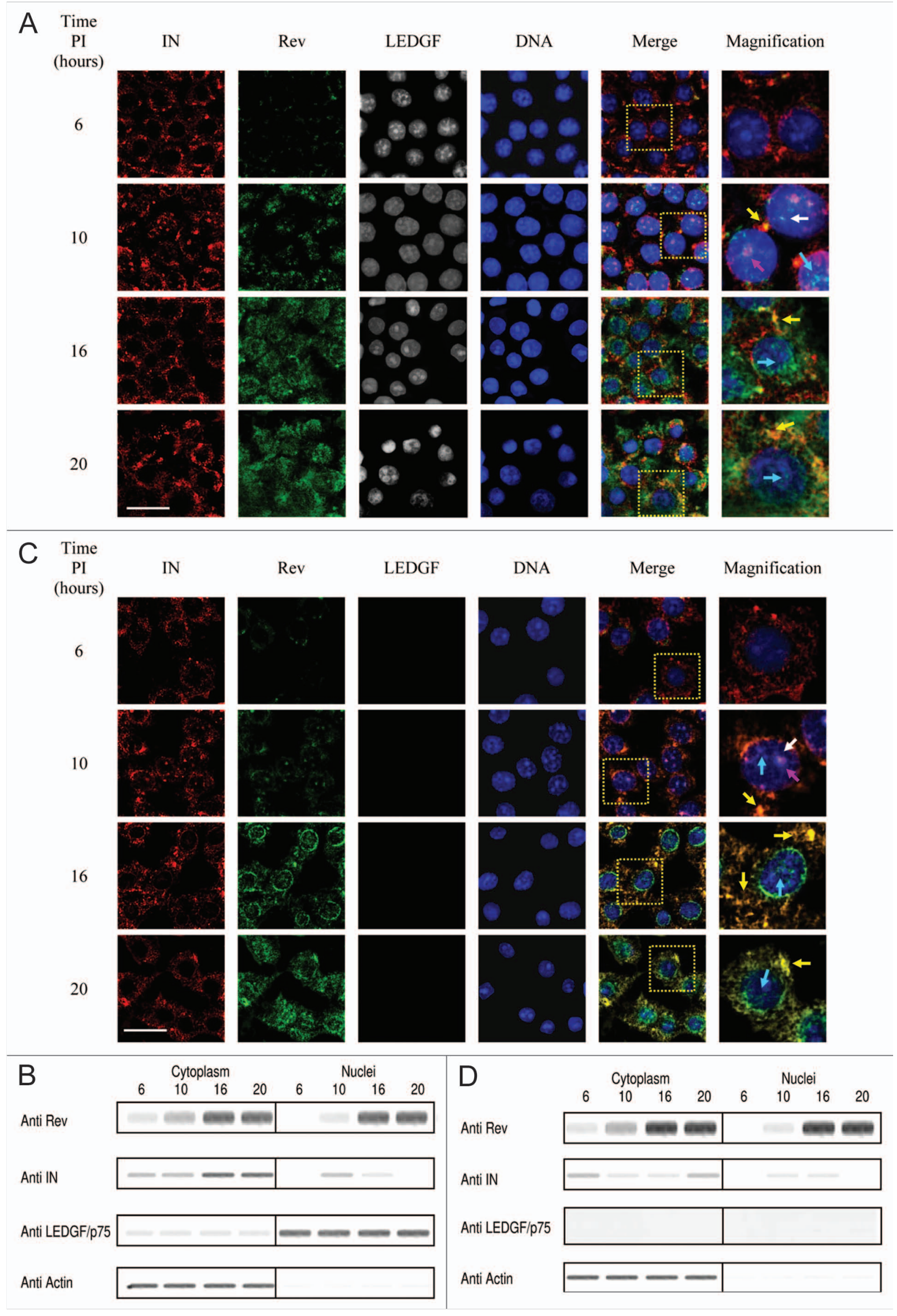


Figure 1 (See opposite page). Transient nuclear localization of HIV-1 IN in virus-infected cells. (A) HeLa-P4 cells were infected with WT HIV-1, and IN (red), Rev (green), LEDGF/p75 (gray) were immunostained and DNA (blue) was stained with DAPI. (B) Sup-T1 cells were infected with WT HIV-1 and the cytoplasmic and nuclear fractions were analyzed by western blot to detect Rev, IN, LEDGF/p75 and actin. (C) Same as (A) but LEDGF/p75-knockdown cells (HeLa-P4/shp75Cl15) were used for infection. (D) Same as in (B) but with the use of LEDGF/p75-knockdown cells (Sup-T1/TL3). In (A and C), scale bar represents $10 \mu \mathrm{m}$. All other experimental details are as described in Materials and Methods. Arrow indicate the presence of intranuclear IN (magenta), Rev (cyan) molecules, Rev-IN complex (white) or cytoplasmic Rev-IN complex (orange-yellow). Areas which have been magnified are marked by yellow boxes.

Inhibition of Rev nuclear export promotes nuclear accumulation of IN. When the HeLa-P4 cells were infected with the Rev M10 HIV mutant, the viral Rev was constrained to the cell's nuclei (Fig. 5A and B). Surprisingly in these cells also IN was exclusively located in the nucleus (Fig. 5A and B). This is in contrast to cells infected by the WT virus, in which IN was only transiently intranuclear (Fig. 1A and B). Intranuclear localization of Rev and IN was also obtained when WT-infected cells were treated with a cell-permeable peptide bearing the Rev NES sequence, which inhibits nuclear export of $\operatorname{Rev}^{31}$ (not shown). The differences observed between the intracellular localization of IN in the Rev M10- and WT-infected cells (see Fig. 1A) may imply that Rev mediates nuclear export of IN. Similarly, intranuclear localization of both IN and Rev was also obtained when nuclear export of Rev was inhibited by leptomycin B in WT-infected cells (Fig. 6A and B). This further supports the assumption that Rev mediates nuclear export of IN. Removal of leptomycin B, which allows nuclear export of Rev, also resulted in nuclear export of IN (Fig. 6C and D). On the other hand, addition of the $\mathrm{INr}$ peptides, which promote dissociation of the Rev-IN complex, ${ }^{20,21}$ prior to and during the leptomycin B wash resulted in nuclear export of only Rev while IN remained intranuclear (Fig. 6C and D). Dissociation of the intranuclear Rev-IN complex by the INr peptides was further supported by the results depicted in Figure 6E: A relatively high degree of integration, about 7 integration events/cell, was obtained following the addition of the INr peptides to the leptomycin B-treated cells compared to 1-2 integration events/cell observed in the absence of the INrs (Fig. 6E).

\section{Discussion}

We previously explained the low degree of integration events observed in HIV-infected cells by the ability of Rev to inhibit IN enzymatic activity following the formation of a Rev-IN complex. ${ }^{20,21}$ Based on these results, we assumed that the Rev-IN complex fails to catalyze the integration reaction in vivo. ${ }^{20}$ Dissociation of the Rev-IN complex by IN-derived peptides abrogated the inhibitory effect of Rev in vitro and stimulated the degree of integration in vivo. ${ }^{21}$

The results of the present work suggest that HIV-1 developed two additional Rev-dependent regulatory steps to ensure low levels of cDNA integration. We show that immediately after infection, Rev inhibits nuclear import of IN (probably bound to the viral DNA within the PIC), thereby reducing the probability of translocation of free IN-DNA complexes into the intranuclear space and thus interaction with the host chromosomal DNA. It is unclear how incoming integrase (virion associated) is differentiated from integrase that is produced after infection from the viral DNA. However, any IN-DNA molecules that escape interaction with the cytoplasmic Rev and are translocated into the host-cell nucleus may then interact with the intranuclear Rev, which promotes dissociation of IN from the cDNA, ${ }^{28}$ inhibits its activity and expels it from the nucleus in the form of Rev-IN complex (see proposed model in Fig. 7). At this stage it is entirely unclear whether IN is exported from the nuclei together with the other PIC components or rather an IN-deficient PIC remains located within the nuclei of the infected cells.

Inhibition of IN nuclear import by Rev is evident from the experiments showing that IN is only cytoplasmic in infected cells treated with Rev-derived peptides or following infection of Revoverexpressing cells. The failure of the Rev-IN complex to penetrate the nuclei of infected cells is probably not due to the size of the complex, which is able to exit the nuclei. Rather, it may be due to mutual masking of the NLSs of both proteins. This can be inferred from studies which elucidated the domain mediating the interaction between these two proteins. ${ }^{21,28}$ Furthermore, nuclear import of both IN and Rev was inhibited when HeLa cells were co-transfected with expression vectors bearing both proteins. The observation that Rev inhibits IN nuclear import is further strengthened by the results showing vast nuclear accumulation of IN in $\Delta$ Rev-infected cells or in cells treated with the INr peptides. The observation that IN is translocated into the nuclei in the presence of the INrs, which promote Rev-IN dissociation, ${ }^{21}$ is not surprising since the IN is considered to be a karyoophilic protein and has even been claimed to mediate nuclear entry of the viral cDNA. ${ }^{12,32-39}$

The involvement of Rev in IN nuclear retention once it is intranuclear should be inferred from our experiments showing preservation of nuclear IN in cells infected with the Rev M10 mutant virus or in leptomycin B-treated cells. It has been well established that in both of these cells, Rev remains intranuclear, being unable to exhibit nuclear export activities. ${ }^{29,30}$ Thus it appears that any Rev-IN complex, similar to the Rev itself, will remain intranuclear in these cells. ${ }^{29,30}$

It is conceivable that due to a certain degree of the Rev-IN complex dissociation, small amount of both Rev and IN are translocated into the nuclei but because of the presence of the Rev NES, Rev-IN complexes formed in the nuclei are exported to the cytoplasm (a possibility demonstrated in the present work). This may indicate that as opposed to the NLS domains, which are presumably masked in the Rev-IN complex, the Rev NES domain is not. In the presence of leptomycin, nuclear retention of Rev results in nuclear accumulation of both Rev and IN as a Rev-IN complex. 

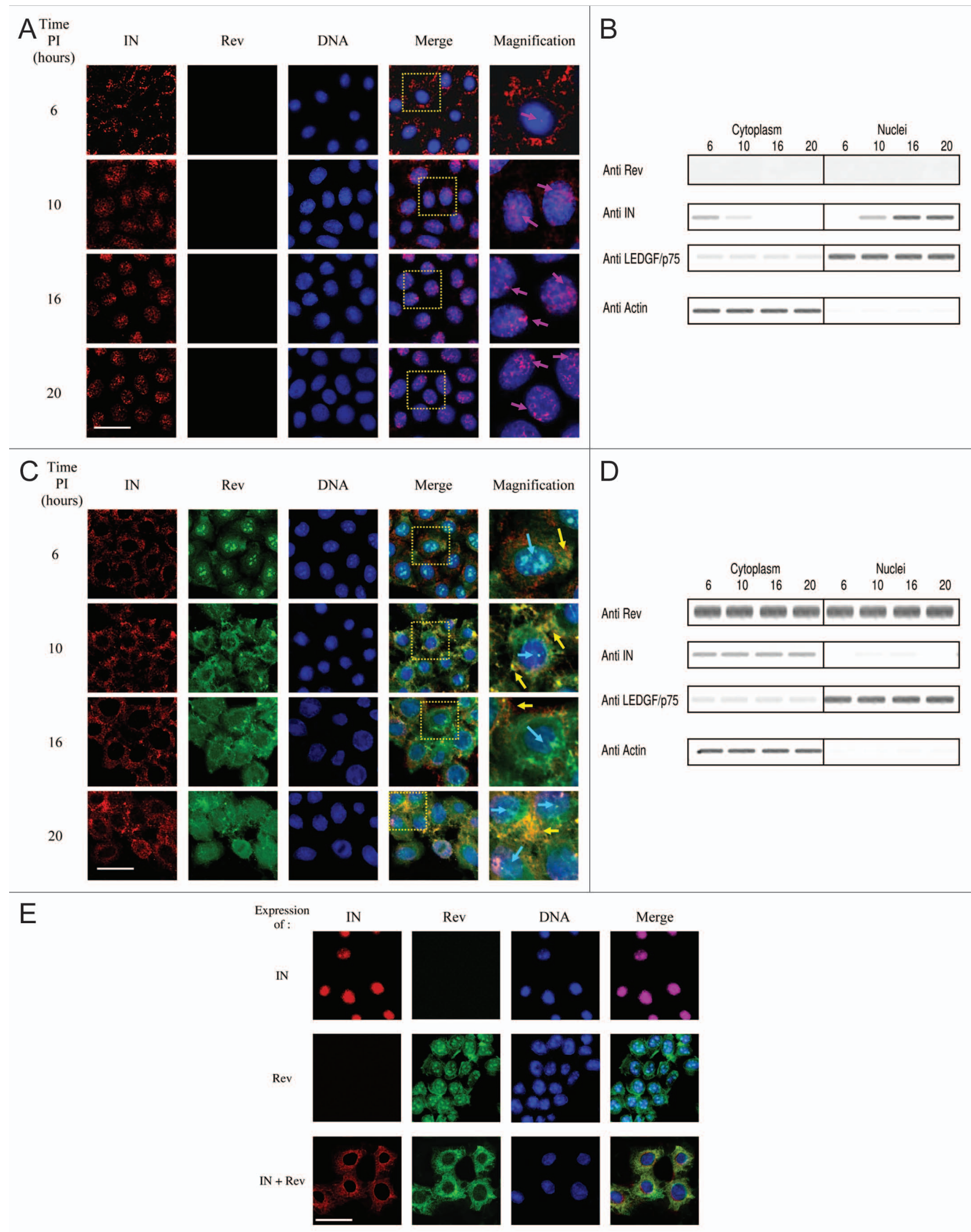
Figure 2 (See opposite page). Rev-induced inhibition of IN nuclear import in HIV-1 infected cells. (A) HeLa-P4 cells were infected with $\Delta$ Rev HIV-1, and IN (red), Rev (green) were immunostained and DNA (blue) was stained with DAPI (B) H9 cells were infected with $\triangle$ Rev HIV-1 and the cytoplasmic and nuclear fractions were analyzed by western blot to detect Rev, IN, LEDGF/p75 and actin. (C) Same as in (A), but Rev-overexpressing HeLa-P4 cells were infected with WT HIV-1. (D) Same as in (B), but Rev-overexpressing H9 cells were infected with WT HIV-1. (E) HeLa-P4 cells were transfected with vectors bearing either the IN, Rev or both as described in Material and Methods. IN (red), Rev (green) were immunostained and DNA (blue) was stained with DAPI. In (A, C and E), scale bar represents $10 \mu \mathrm{m}$. All other experimental details are as described in Materials and Methods. Arrow indicate the presence of intranuclear IN (magenta), Rev (cyan) molecules or cytoplasmic Rev-IN complex (orange-yellow). Areas which have been magnified are marked by yellow boxes.
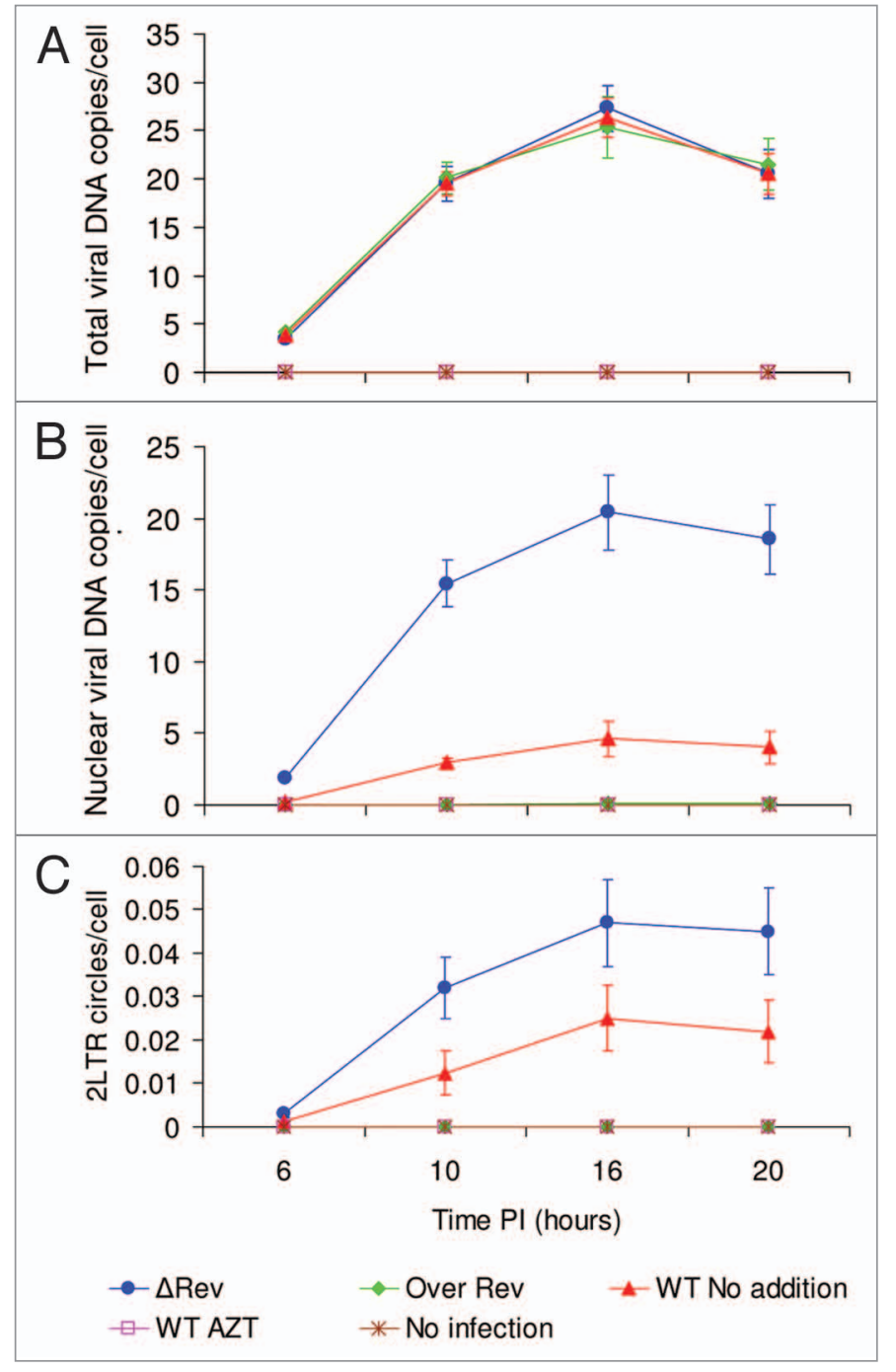

Figure 3. Rev-induced inhibition of viral DNA nuclear import. Revoverexpressing $\mathrm{H} 9$ cells were infected with WT HIV-1 ( ), or H9 cells were infected with $\triangle$ Rev HIV-1 ( ), wt HIV-1 ( ), WT HIV-1 with $2 \mu$ M AZT ( ) or not infected $(*)$ and the amount of total viral DNA $(A)$, nuclear viral DNA (B) and 2LTR circles (C) was estimated at different times PI as described in Materials and Methods. Bars represent deviations between the three repeated experiments.

Our observation that nuclear accumulation of IN is transient and no intranuclear IN is present in late stages of infection is somewhat unexpected since no NESs have been been identified within its sequence. Furthermore, in cultured cells expressing only IN, it accumulated within the nuclei with no indication of its ability to undergo nuclear export. ${ }^{40}$ The view that Rev mediates nuclear export of the intranuclear IN as part of a Rev-IN complex is supported by the experiments showing translocation of both into the cytoplasm following removal of leptomycin B. This view is further supported by the results showing that following disruption of the intranuclear Rev-IN complex by the INr peptides, concomitant with removal of leptomycin B, Rev is exported from the nuclei while the IN, lacking an NES sequence, remains intranuclear and therefore is able to stimulate the integration reaction. In summary, it appears that together with our previous observations, ${ }^{20,21,28}$ the present results convert the Rev protein from an accessory viral protein ${ }^{22}$ to a major regulator. Thus, according to our results, Rev controls crucial steps during both the early cDNA integration and the late viral RNA nuclear export stages of the HIV life cycle.

\section{Materials and Methods}

Cells. Monolayer adherent HEK293T cells, HeLa-P4 cells overexpressing Rev, IN or both and HeLa-P4 cells were grown in Dulbecco's modified Eagle's medium (DMEM). The T-lymphocyte cell lines Sup-T1, H9 and Rev-overexpressing H9 were grown in RPMI 1640 medium. Cells other than the Revoverexpressing cells were provided by the NIH Reagent Program, Division of AIDS, NIAID, NIH (Bethesda, MD). Cells were incubated at $37^{\circ} \mathrm{C}$ in a $5 \% \mathrm{CO}_{2}$ atmosphere. All media were supplemented with $10 \%(\mathrm{v} / \mathrm{v})$ fetal calf serum, $0.3 \mathrm{~g} / \mathrm{l} \mathrm{L}$-glutamine, $100 \mathrm{U} / \mathrm{ml}$ penicillin and $100 \mathrm{U} / \mathrm{ml}$ streptomycin (Biological Industries, Beit Haemek, Israel). HeLa-P4/shp75Cl15 cells, a generous gift from Prof. Z. Debyser (Molecular Medicine, K.U. Leuven, Flanders, Belgium), were grown as described in. ${ }^{41}$ SupT1/TL3 (LEDGF/p75 knockdown) cells, a generous gift from Prof. Poeschla (Department of Molecular Medicine, Mayo Foundation, Rochester $\mathrm{MN}$ ), were grown as described previously. ${ }^{42}$ Rev/IN-overexpressing cells were generated by transfection into HeLa-P4 cells ${ }^{43}$ with pcDNA3.1 plasmid bearing the full Rev, IN or both.

Viruses. WT HIV-1 (HXB2 $\left.{ }^{44}\right)$, as well as the IN mutant D64N D116N, 45 were generated by transfection into HEK293T cells ${ }^{43}$ of the virus-containing plasmid. $\Delta$ Rev pLAIY47H2, ${ }^{46}$ and Rev M10, ${ }^{29}$ HIVs were generated by transfection into Rev-overexpressing cells. Viruses were harvested and stored as described in. ${ }^{21}$ The pLAIY47H2,${ }^{46}$ viruses were a generous gift from Prof. B. Berkhout (Department of Human Retrovirology, Academic Medical Center, University of Amsterdam, The Netherlands), and the IN mutant D64N D116N virus was a generous gift from Prof. A. Engelman (Department of Cancer Immunology and AIDS Dana-Farber 


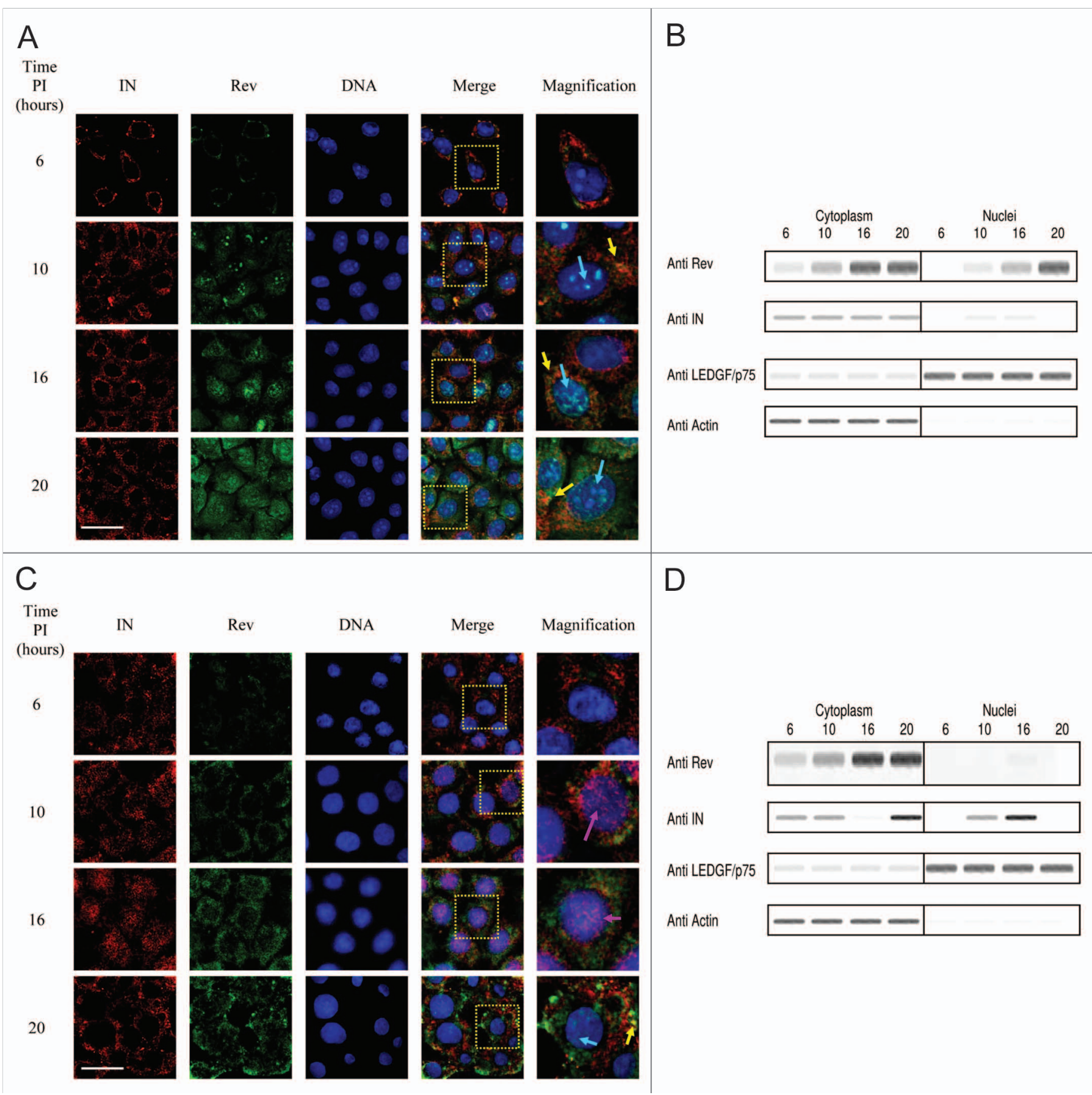

Figure 4. Effect of the Rev- and IN-derived peptides on nuclear localization of viral IN. (A) HeLa-P4 cells were infected with WT HIV-1 in the presence of Rev 13-23 peptide, and IN (red), Rev (green) were immunostained and DNA (blue) was stained with DAPI (B) H9 cells were infected with WT HIV-1 in the presence of Rev 13-23 peptide and the cytoplasmic and nuclear fractions were analyzed by western blot to detect Rev, IN, LEDGF/p75 and actin. $(C$ and $D)$, same as in (A and B), respectively, but in the presence of $\mathrm{INr}-1$. In ( $A$ and $C$ ), scale bar represents $10 \mu \mathrm{m}$. All other experimental details are as described in Materials and Methods. Arrow indicate the presence of intranuclear IN (magenta), Rev (cyan) molecules or cytoplasmic Rev-IN complex (orange-yellow). Areas which have been magnified are marked by yellow boxes.

Cancer Institute and Division of AIDS, Harvard Medical School, Boston, MA).

Virus stock titration and normalization. Quantitative titration of HIV-1 was carried out using the MAGI assay, as described. ${ }^{47}$ Briefly, TZM-b1 cells were grown in 96-well plates at $1 \times 10^{4}$ cells per well. The cells were infected with $50 \mu \mathrm{l}$ of serially diluted virus (wild-type, $\Delta$ Rev or Rev M10 HIV-1) as described. ${ }^{47}$ Two days post-infection (PI), cultured cells were fixed and $\beta$-galactosidase was estimated exactly as described previously. ${ }^{47}$ Blue cells were counted under a light microscope at 200X magnification. Also the amount of viral RNA was estimated by real time reverse transcription PCR as described in. ${ }^{48}$ 


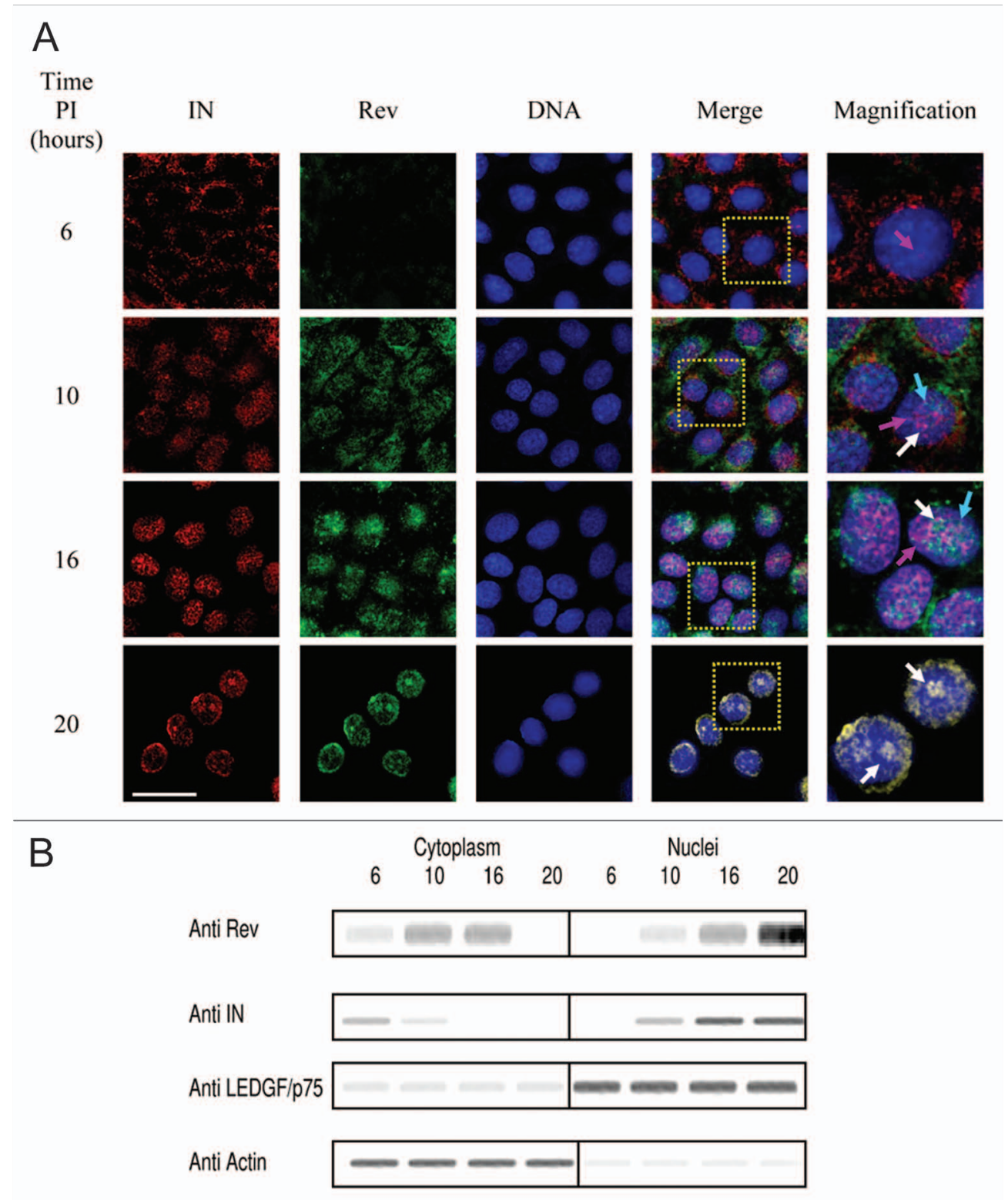

Figure 5. Nuclear localization of viral IN and Rev in Rev M10 HIV-1-infected cells. (A) HeLa-P4 cells were infected with Rev M10 HIV-1, and IN (red), Rev (green) were immunostained and DNA (blue) was stained with DAPI. Scale bar represents $10 \mu \mathrm{m}$. (B) H9 cells were infected with Rev M10 HIV-1 and the cytoplasmic and nuclear fractions were analyzed by western blot to detect Rev, IN, LEDGF/p75 and actin. All other experimental details are as described in Materials and Methods. Arrow indicate the presence of intranuclear IN (magenta), Rev (cyan) molecules, Rev-IN complex (white) or cytoplasmic Rev-IN complex (orange-yellow). Areas which have been magnified are marked by yellow boxes.

Infection of cultured cells. Cultured lymphocytes were infected exactly as described. ${ }^{21}$ Briefly, cultured lymphocytes $\left(1 \mathrm{x} 10^{5}\right)$ were centrifuged for $5 \mathrm{~min}$ at $2,000 \mathrm{rpm}$ and after removal of the supernatant the cells were resuspended in 0.2 to $0.5 \mathrm{ml}$ of RPMI 1640 medium containing virus at the indicated multiplicity of infection (MOI). Following absorption for $2 \mathrm{~h}$ at $37^{\circ} \mathrm{C}$, the cells were washed to remove unbound virus and then incubated at the same temperature in RPMI 1640 medium. Cultured monolayer cells (HeLa-P4, Rev overexpressing cells and HeLa-P4/shp75Cl15 cells) were grown for $24 \mathrm{~h}$ before infection, then the medium was discarded and cells were incubated at different multiplicity of infections (MOI) with the indicated virus for $2 \mathrm{~h}$ at $37^{\circ} \mathrm{C}$. Cells were washed three times with PBS and incubated in DMEM.

Peptide synthesis and purification. Peptides were synthesized on an Applied Biosystems (ABI) 433A peptide synthesizer and purification was performed in a Gilson HPLC using a reversephase C8 semi-preparative column (ACE, Advanced Chromatography Technologies, USA) as described previously. ${ }^{20,49}$

Study of cellular localization by western blot. Cells were infected at MOI of 15 for the indicated viruses. Cells were 


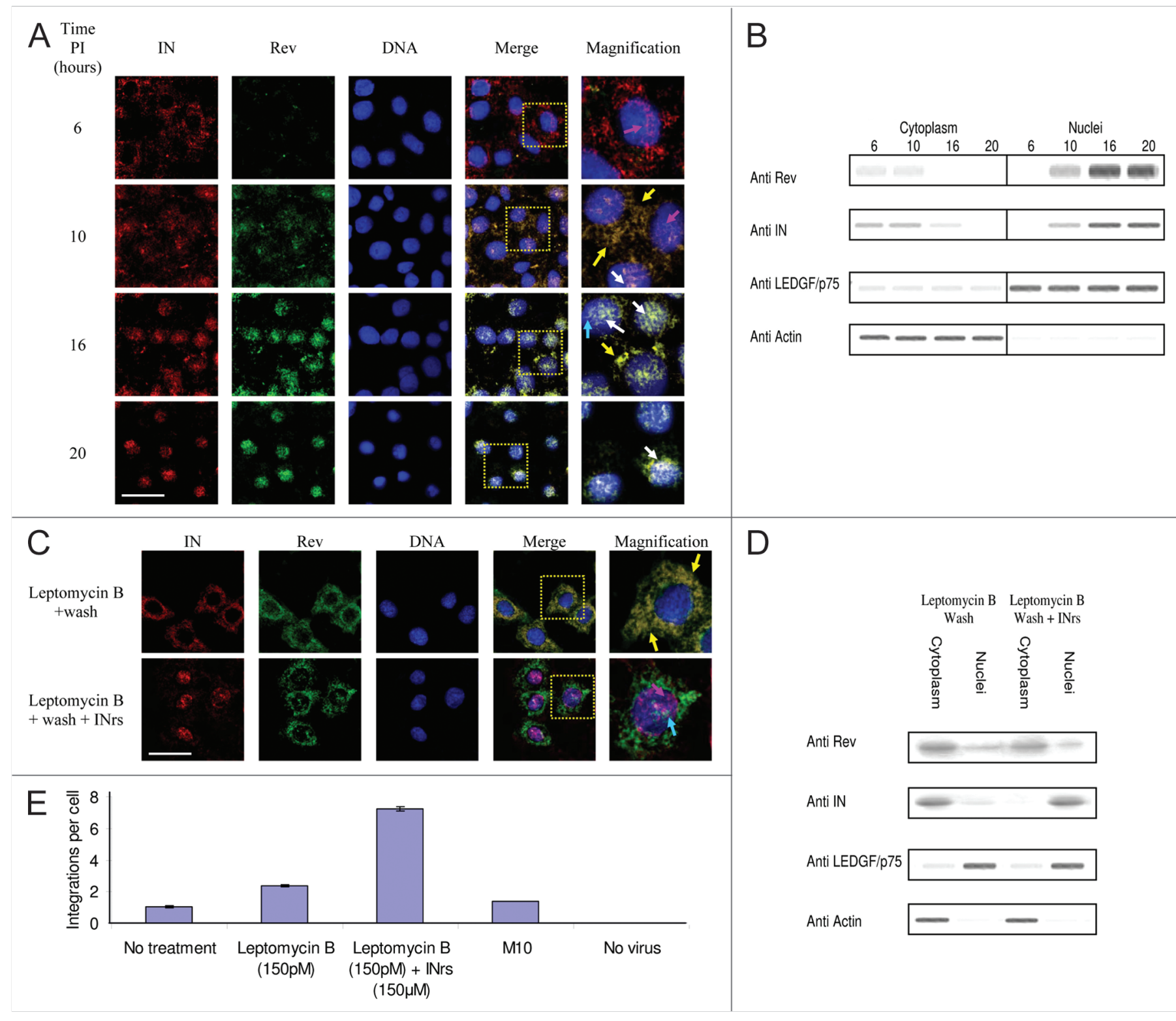

Figure 6. Rev promotes nuclear export of viral IN: effect of the INr peptide. (A) HeLa-P4 cells were infected with WT HIV-1 in the presence of leptomycin B, and IN (red), Rev (green) were immunostained and DNA (blue) was stained with DAPI. (B) H9 cells were infected with WT HIV-1 in the presence of leptomycin B and the cytoplasmic and nuclear fractions were analyzed by western blot. (C) Same as in (A) but the leptomycin B was washed out by three washes with PBS (with or without the two INr peptides) and new medium was added with or without INr peptides. (D) Same as in (C) but the cytoplasmic and nuclear fractions were analyzed by western blot. (E) The number of integration events per cell was estimated in $\mathrm{H} 9$ cells infected with WT or M10 HIV following the indicated treatment. In (A and C), scale bar represents $10 \mu \mathrm{m}$. All other experimental details are as described in Materials and Methods. Arrow indicate the presence of intranuclear IN (magenta), Rev (cyan) molecules, Rev-IN complex (white) or cytoplasmic Rev-IN complex (orange-yellow). Areas which have been magnified are marked by yellow boxes.

harvested at different times PI, washed three times in PBS and lysed by the addition of PBS containing $1 \%(\mathrm{v} / \mathrm{v})$ Triton X-100 for whole-cell lysate, or cytoplasm and nuclei were isolated as described below. The lysate or the isolated fractions were subjected to SDS-PAGE and immunoblotted with a monoclonal antiRev antibody $(\alpha-R e v),{ }^{50}$ antiserum raised against IN amino acids 276-288 ( $\alpha$-IN) (NIH AIDS Research \& Reference Reagent Program catalog number 758 ), anti-LEDGF/p75 ( $\alpha$-LEDGF/ p75) (R\&D Systems) or anti-actin ( $\alpha$-actin) antibody (Santa Cruz), and the complementary HRP-conjugated secondary antibodies (Jackson). When peptides were used, cells were incubated with $150 \mu \mathrm{M}$ of the indicated peptide for $2 \mathrm{~h}$ prior to infection. Leptomycin B was used at $150 \mathrm{pM}$. Quantitative estimation of the bands was performed by Image Gauge V3.46 software (Fujifilm) (See Tables S1 and S2).

Isolation of cytoplasm and nuclei from infected cells. The various fractions were obtained from virus-infected cells essentially as previously described ${ }^{51}$ with several modifications. Briefly, cells were harvested and washed twice in buffer A ( $20 \mathrm{mM}$ Hepes $\mathrm{pH}$ 7.3, $150 \mathrm{mM} \mathrm{KCl}, 5 \mathrm{mM} \mathrm{MgCl}, 1 \mathrm{mM}$ DTT and $0.1 \mathrm{mM}$ 


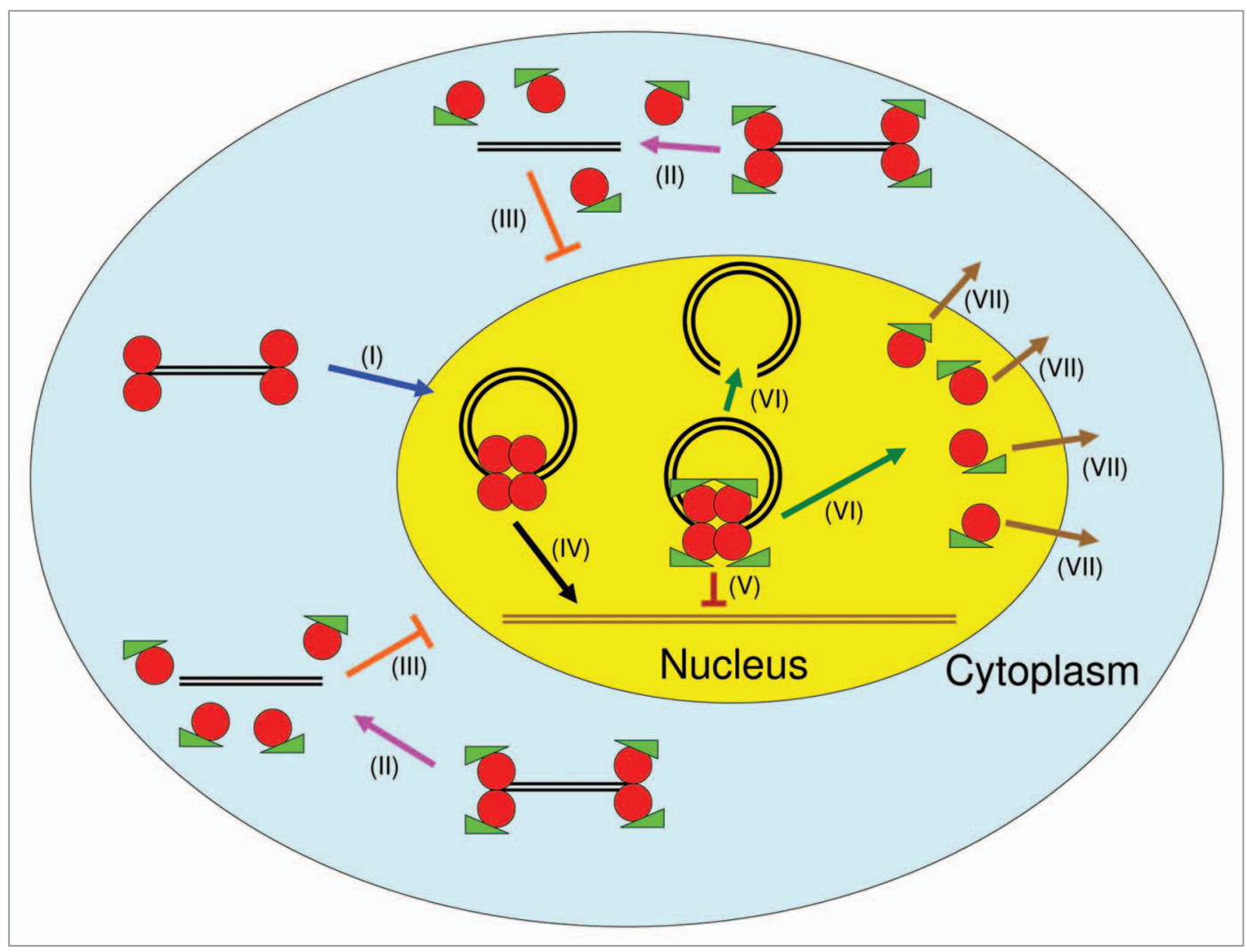

Figure 7. A schematic model explaining the involvement of Rev in nucleocytoplasmic trafficking of IN in HIV-1 infected cells. In the cytoplasmic pre-integration complex, the IN molecules (red circle) are bound to viral DNA (double black lines) resulting in the formation of an IN-DNA complex. A certain amounts of the IN-DNA complexes are translocated into the nucleus (blue arrow) (I) (presumably up to $10 \mathrm{~h} \mathrm{PI}$ ) before sufficient amount of Rev molecules are synthesized. Binding of Rev molecules (green triangle) to the IN induces dissociation of the IN-DNA complex (magenta arrow) (II) and blocks nuclear import (orange blocked arrow) of the IN (III) (from $6 \mathrm{~h} \mathrm{PI).} \mathrm{Within} \mathrm{the} \mathrm{nucleus,} \mathrm{the} \mathrm{IN} \mathrm{is} \mathrm{able} \mathrm{to} \mathrm{mediate} \mathrm{integration} \mathrm{of} \mathrm{only} \mathrm{one} \mathrm{or} \mathrm{two}$ viral DNA molecules (black arrow) into the host genome (double brown line) (IV) (10-16 h PI). The IN of the remaining IN-DNA complexes interacts with the intranuclear Rev, thus blocking its enzymatic activity and consequently the integration process (red blocked arrow) (V). Similar to the cytoplasmic Rev also the intranuclear Rev molecules promote dissociation of the IN-DNA complex (green arrow) (VI) and mediate nuclear export of the free IN (brown arrow) (VII) (from $16 \mathrm{~h} \mathrm{PI).}$

PMSF). Cells were then suspended in $200 \mu \mathrm{l}$ of buffer A with $0.025 \%(\mathrm{w} / \mathrm{v})$ digitonin and incubated at room temperature for $10 \mathrm{~min}$. Cells were centrifuged for $3 \mathrm{~min}$ at $1,000 \mathrm{~g}$ at room temperature. The supernatant was then centrifuged at $8,000 \mathrm{~g}$ and separated into supernatant (cytoplasm) and pellet (nuclei) and stored at $-70^{\circ} \mathrm{C}$.

Quantitative analysis of copy numbers of HIV-1 DNA integrated into the cellular genome. The integration reaction, as well as the integration events, were performed exactly as described previously. ${ }^{21}$ Briefly, Integrated HIV-1 sequences were amplified by two PCR replication steps using the HIV-1 LTR-specific primer (LTR-TAG-F 5'-ATG CCA CGT AAG CGA AAC TCT GGC TAA CTA GGG AAC CCA CTG-3') and Alu-targeting primers (first-Alu-F 5'-AGC CTC CCG AGT AGC TGG GA-3' and first-Alu-R 5'-TTA CAG GCA TGA GCC ACC G-3').52 Alu-LTR fragments were amplified from $10 \mathrm{ng}$ of total cell DNA in a $25-\mu$ reaction mixture containing $1 \mathrm{X}$ PCR buffer, $3.5 \mathrm{mM}$ $\mathrm{MgCl}_{2}, 200 \mu \mathrm{M}$ dNTPs, $300 \mathrm{nM}$ primers, and 0.025 units $/ \mu \mathrm{l}$ of Taq polymerase. The first-round PCR cycle conditions were as follows: a DNA denaturation and polymerase activation step of $10 \mathrm{~min}$ at $95^{\circ} \mathrm{C}$ and then 12 cycles of amplification $\left(95^{\circ} \mathrm{C}\right.$ for $15 \mathrm{~s}, 60^{\circ} \mathrm{C}$ for $30 \mathrm{~s}, 72^{\circ} \mathrm{C}$ for $5 \mathrm{~min}$ ). During the secondround PCR, the first-round PCR product could be specifically amplified by using the tag-specific primer (tag-F 5'-ATG CCA CGT AAG CGA AAC TC-3') and the LTR primer (LTR-R 5'-AGG CAA GCT TTA TTG AGG CTT AAG-3') designed by PrimerExpress (Applied Biosystems) using default settings. The second-round PCR was performed on $1 / 25^{\text {th }}$ of the firstround PCR product in a mixture containing $300 \mathrm{nM}$ of each primer, $12.5 \mu \mathrm{l}$ of $2 \mathrm{X}$ SYBR Green master mixture (Applied Biosystems) at a final volume of $25 \mu \mathrm{l}$, run on an ABI PRIZM 7700 (Applied Biosystems). The second-round PCR cycles began with DNA denaturation and a polymerase-activation step $\left(95^{\circ} \mathrm{C}\right.$ for $10 \mathrm{~min})$, followed by 40 cycles of amplification $\left(95^{\circ} \mathrm{C}\right.$ for $15 \mathrm{~s}, 60^{\circ} \mathrm{C}$ for $\left.60 \mathrm{~s}\right)$.

For generation of a standard calibration curve, the SVC21 plasmid containing the full-length $\mathrm{HIV}-1_{\mathrm{HXB} 2}$ viral DNA was used as a template. In the first-round PCR, the LTR-TAG-F and LTR-R primers were used and the second-round PCR was performed using the tag-F and LTR-R primers. The standard linear curve was in the range of $5 \mathrm{ng}$ to $0.25 \mathrm{fg}(R=0.99)$. DNA samples were assayed with quadruplets of each sample. For further 
experimental details see. ${ }^{28}$ The cell equivalents in the sample DNA were calculated based on amplification of the $18 \mathrm{~S}$ gene by real-time PCR as described. ${ }^{53}$

Quantification of total and nuclear viral DNA. Total viral DNA was estimated using SYBR green real-time quantitative PCR at the indicated times PI and from the total or nuclear isolated fraction of the infected cells. All other details were exactly as previously described.${ }^{54}$ Briefly, DNA samples (1 $\mu \mathrm{g}$ of DNA) were added to $95 \mu \mathrm{l}$ containing 1x Hot-Rescue Real Time PCR Kit-SG (Diatheva s.r.l, Fano, Italy), and $100 \mathrm{nM}$ of each PBS (primer-binding site) primer: F5 (5' primer, 5'-TAG CAG TGG CGC CCG A-3') and R5 (3' primer, 5'-TCT CTC TCC TTC TAG CCT CCG C-3'). All amplification reactions were carried out using an ABI Prism 7700 Sequence Detection System (Applied Biosystems): One cycle at $95^{\circ} \mathrm{C}$ for $10 \mathrm{~min}$, followed by 45 cycles of $15 s$ at $95^{\circ} \mathrm{C}$ and $35 s$ at $68^{\circ} \mathrm{C}$. In each PCR run, three replicates were performed.

Quantification of 2LTR circles. Quantification of 2LTR circles was performed exactly as previously described. ${ }^{1}$

Immunostaining. Cells were grown on chamber slides (Nunc) then infected with the indicated HIV-1 at a MOI of 25. Cells were fixed at different times PI exactly as described ${ }^{55}$ and immunostained essentially as described ${ }^{55}$ with some modifications. Briefly, after fixation, cells were blocked with 5\% (w/v) IgG-free BSA (Jackson) in PBS for 60 min. For detection of HIV-1 IN and Rev and the host LEDGF/p75, the cells were incubated with 1:50 rabbit $\alpha$-IN(NIH AIDS Research \& Reference Reagent Program catalog number 758), 1:50 rat $\alpha-\operatorname{Rev}^{50}$ and 1:100 goat $\alpha$-LEDGF/p75 (R\&D Systems) at room temperature for $60 \mathrm{~min}$ each. Cells were washed five times with PBS $+0.05 \%$ (v/v) Tween 20 between antibodies. Then the cells were incubated with the respective secondary antibodies: Cy3-conjugated anti-rabbit, Cy2-conjugated anti-rat and Cy5-conjugated anti-goat (Jackson) (all diluted 1:100) at room temperature for $60 \mathrm{~min}$ each, with five washes with PBS $+0.05 \%$ Tween 20 between antibodies. For detection of DNA, cells were stained with DAPI according to the manufacturer's protocol. Slides were prepared with Mounting Media (Bio-Rad) and immunofluorescent cells were detected with an Olympus confocal microscope.

To draw comprehensible conclusions from our microscopic observations, several steps had to be taken to better visualize the labeled viral proteins: (1) Cells were infected with a very high titer of virus (MOI of 25). (2) Relatively high concentrations of antibodies were used. (3) For detection of immunolabeled proteins, slides were overexposed. For clarification, the same samples following the high exposure (Figs. S1 and S2) and original low exposure (Figs. S3 and S4) have been added to the supplementary data.

All of the experiments described in the present work were repeated at least three times.

\section{Acknowledgements}

This work was supported by the Israeli Science Foundation (A. Loyter) and by a starting grant from the European Research Council (ERC) (to A.F.). The authors are imbedded to Prof. Z. Debyser (Molecular Medicine, K.U. Leuven, Flanders, Belgium) and Prof. Poeschla (Department of Molecular Medicine, Mayo Foundation, Rochester MN) for the LEDGF/p75 knockdown cells. The authors also imbedded to Prof. B. Berkhout (Department of Human Retrovirology, Academic Medical Center, University of Amsterdam, The Netherlands) and Prof. A. Engelman (Department of Cancer Immunology and AIDS Dana-Farber Cancer Institute and Division of AIDS, Harvard Medical School, Boston, MA) for the mutated viral strands.

\section{Note}

Supplementary materials can be found at:

www.landesbioscience.com/supplement/LevinNUC1-2-Sup.pdf

\section{References}

1. Butler SL, Hansen MS, Bushman FD. A quantitative assay for HIV DNA integration in vivo. Nat Med 2001; 7:631-4.

2. Chun TW, Carruth L, Finzi D, Shen X, DiGiuseppe JA, Taylor H, et al. Quantification of latent tissue reservoirs and total body viral load in HIV-1 infection. Nature 1997; 387:183-8.

3. Esposito D, Craigie R. HIV integrase structure and function. Adv Virus Res 1999; 52:319-33.

4. Pommier Y, Johnson AA, Marchand C. Integrase inhibitors to treat HIV/AIDS. Nat Rev Drug Discov 2005; 4:236-48.

5. Bukrinsky MI, Sharova N, McDonald TL, Pushkarskaya T, Tarpley WG, Stevenson M. Association of integrase, matrix and reverse transcriptase antigens of human immunodeficiency virus type 1 with viral nucleic acids following acute infection. Proc Natl Acad Sci USA 1993; 90:6125-9.

6. Engelman A, Mizuuchi K, Craigie R. HIV-1 DNA integration: mechanism of viral DNA cleavage and DNA strand transfer. Cell 1991; 67:1211-21.

7. Guiot E, Carayon K, Delelis O, Simon F, Tauc P, Zubin $\mathrm{E}$, et al. Relationship between the oligomeric status of HIV-1 integrase on DNA and enzymatic activity. J Biol Chem 2006; 281:22707-19.
8. Chen A, Weber IT, Harrison RW, Leis J. Identification of amino acids in HIV-1 and avian sarcoma virus integrase subsites required for specific recognition of the long terminal repeat Ends. J Biol Chem 2006; 281:4173-82.

9. Heuer TS, Brown PO. Photo-cross-linking studies suggest a model for the architecture of an active human immunodeficiency virus type 1 integrase-DNA complex. Biochemistry 1998; 37:6667-78.

10. Van Maele B, Busschots K, Vandekerckhove L, Christ F, Debyser Z. Cellular co-factors of HIV-1 integration. Trends Biochem Sci 2006; 31:98-105.

11. Depienne C, Roques P, Creminon C, Fritsch L, Casseron R, Dormont D, et al. Cellular distribution and karyophilic properties of matrix, integrase and $\mathrm{Vpr}$ proteins from the human and simian immunodeficiency viruses. Exp Cell Res 2000; 260:387-95.

12. Gallay P, Hope T, Chin D, Trono D. HIV-1 infection of nondividing cells through the recognition of integrase by the importin/karyopherin pathway. Proc Natl Acad Sci USA 1997; 94:9825-30.

13. Haffar OK, Popov S, Dubrovsky L, Agostini I, Tang $\mathrm{H}$, Pushkarsky T, et al. Two nuclear localization signals in the HIV-1 matrix protein regulate nuclear import of the HIV-1 pre-integration complex. J Mol Biol 2000; 299:359-68.

14. Jenkins $\mathrm{Y}$, McEntee M, Weis K, Greene WC. Characterization of HIV-1 vpr nuclear import: analysis of signals and pathways. J Cell Biol 1998; 143:875-85.
15. Reil H, Bukovsky AA, Gelderblom HR, Gottlinger HG. Efficient HIV-1 replication can occur in the absence of the viral matrix protein. EMBO J 1998; 17:2699-708.

16. Sherman MP, Greene WC. Slipping through the door: HIV entry into the nucleus. Microbes Infect 2002; 4:67-73.

17. Yamashita M, Emerman M. The cell cycle independence of HIV infections is not determined by known karyophilic viral elements. PLoS Pathog 2005; 1:18.

18. Zennou V, Petit C, Guetard D, Nerhbass U, Montagnie L, Charneau P. HIV-1 genome nuclear import is mediated by a central DNA flap. Cell 2000; 101:173-85.

19. Yamashita M, Emerman M. Retroviral infection of non-dividing cells: old and new perspectives. Virology 2006; 344:88-93.

20. Levin A, Hayouka Z, Brack-Werner R, Volsky DJ, Friedler A, Loyter A. Novel regulation of HIV-1 replication and pathogenicity: Rev inhibition of integration. Protein Eng Des Sel 2009; 22:753-63.

21. Levin A, Hayouka Z, Helfer M, Brack-Werner R, Friedler A, Loyter A. Peptides derived from HIV-1 integrase that bind Rev stimulate viral genome integration. PLoS ONE 2009; 4:4155.

22. Pollard VW, Malim MH. The HIV-1 Rev protein. Annu Rev Microbiol 1998; 52:491-532.

23. Freed EO. HIV-1 replication. Somat Cell Mol Genet $2001 ; 26: 13-33$. 
24. Iyer SR, Yu D, Biancotto A, Margolis LB, Wu Y. Measurement of HIV-1 preintegration transcription using the Rev-dependent Rev-CEM cell reveals a sizable transcribing DNA population comparable with proviral templates. J Virol 2009; 83:8662-73.

25. Wu Y. HIV-1 gene expression: lessons from provirus and non-integrated DNA. Retrovirology 2004; 1:13.

26. Wu Y, Marsh JW. Early transcription from nonintegrated DNA in human immunodeficiency virus infection. J Virol 2003; 77:10376-82.

27. Levin A, Rosenbluh J, Hayouka Z, Friedler A, Loyter A. Integration of HIV-1 DNA is regulated by interplay between viral Rev and cellular LEDGF/p75 proteins. Mol Med 2010; 16:34-44.

28. Rosenbluh J, Hayouka Z, Loya S, Levin A, ArmonOmer A, Britan E, et al. Interaction between HIV-1 Rev and integrase proteins: a basis for the development of anti-HIV peptides. J Biol Chem 2007; 282:15743-53.

29. Bahner I, Zhou C, Yu XJ, Hao QL, Guatelli JC, Kohn DB. Comparison of trans-dominant inhibitory mutant human immunodeficiency virus type 1 genes expressed by retroviral vectors in human T lymphocytes. J Virol 1993; 67:3199-207.

30. Wolff B, Sanglier JJ, Wang Y. Leptomycin B is an inhibitor of nuclear export: inhibition of nucleo-cytoplasmic translocation of the human immunodeficiency virus type 1 (HIV-1) Rev protein and Rev-dependent mRNA. Chem Biol 1997; 4:139-47.

31. Pasquinelli AE, Powers MA, Lund E, Forbes D, Dahlberg JE. Inhibition of mRNA export in vertebrate cells by nuclear export signal conjugates. Proc Natl Acad Sci USA 1997; 94:14394-9.

32. Armon-Omer A, Graessmann A, Loyter A. A synthetic peptide bearing the HIV-1 integrase 161-173 amino acid residues mediates active nuclear import and binding to importin alpha: characterization of a functional nuclear localization signal. J Mol Biol 2004; 336:111728.

33. Bouyac-Bertoia M, Dvorin JD, Fouchier RA, Jenkins Y, Meyer BE, Wu LI, et al. HIV-1 infection requires a functional integrase NLS. Mol Cell 2001; 7:1025-35.

34. Depienne C, Mousnier A, Leh H, Le Rouzic E, Dormont D, Benichou S, et al. Characterization of the nuclear import pathway for HIV-1 integrase. J Biol Chem 2001; 276:18102-7.

35. Dvorin JD, Bell P, Maul GG, Yamashita M, Emerman M, Malim MH. Reassessment of the roles of integrase and the central DNA flap in human immunodeficiency virus type 1 nuclear import. J Virol 2002; 76:1208796.
36. Ikeda T, Nishitsuji H, Zhou X, Nara N, Ohash T, Kannagi M, et al. Evaluation of the functional involvement of human immunodeficiency virus type 1 integrase in nuclear import of viral cDNA during acute infection. J Virol 2004; 78:11563-73.

37. Limon A, Devroe E, Lu R, Ghory HZ, Silver PA, Engelman A. Nuclear localization of human immunodeficiency virus type 1 preintegration complexes (PICs): V165A and R166A are pleiotropic integrase mutants primarily defective for integration, not PIC nuclear import. J Virol 2002; 76:10598-607.

38. Pluymers W, Cherepanov P, Schols D, De Clercq E, Debyser Z. Nuclear localization of human immunodeficiency virus type 1 integrase expressed as a fusion protein with green fluorescent protein. Virology 1999; 258:327-32.

39. Tsurutani N, Kubo M, Maeda Y, Ohashi T, Yamamoto $\mathrm{N}$, Kannagi $\mathrm{M}$, et al. Identification of critical amino acid residues in human immunodeficiency virus type 1 IN required for efficient proviral DNA formation a steps prior to integration in dividing and nondividing cells. J Virol 2000; 74:4795-806.

40. Maertens G, Cherepanov P, Pluymers W, Busschots K, De Clercq E, Debyser Z, et al. LEDGF/p75 is essentia for nuclear and chromosomal targeting of HIV-1 integrase in human cells. J Biol Chem 2003; 278:3352839.

41. Vandekerckhove L, Christ F, Van Maele B, De Rijck J, Gijsbers R, Van den Haute C, et al. Transient and stable knockdown of the integrase cofactor LEDGF/ p75 reveals its role in the replication cycle of human immunodeficiency virus. J Virol 2006; 80:1886-96.

42. Llano M, Saenz DT, Meehan A, Wongthida P, Peretz $\mathrm{M}$, Walker WH, et al. An essential role for LEDGF/ p75 in HIV integration. Science 2006; 314:461-4.

43. Cullen BR. Use of eukaryotic expression technology in the functional analysis of cloned genes. Methods Enzymol 1987; 152:684-704

44. Ratner L, Haseltine W, Patarca R, Livak KJ, Starcich B, Josephs SF, et al. Complete nucleotide sequence of the AIDS virus, HTLV-III. Nature 1985; 313:277-84.

45. Nakajima N, Lu R, Engelman A. Human immunodeficiency virus type 1 replication in the absence of integrase-mediated dna recombination: definition of permissive and nonpermissive T-cell lines. J Virol 2001; 75:7944-55.
46. Verhoef K, Koper M, Berkhout B. Determination of the minimal amount of Tat activity required for human immunodeficiency virus type 1 replication. Virology 1997; 237:228-36.

47. Kimpton J, Emerman M. Detection of replicationcompetent and pseudotyped human immunodeficiency virus with a sensitive cell line on the basis of activation of an integrated beta-galactosidase gene. J Virol 1992; 66:2232-9.

48. Pizzato $M$, Erlwein $O$, Bonsall D, Kaye $S$, Mui D, McClure MO. A one-step SYBR Green I-based product-enhanced reverse transcriptase assay for the quantitation of retroviruses in cell culture supernatants. J Virol Methods 2009; 156:1-7.

49. Hayouka Z, Rosenbluh J, Levin A, Loya S, Lebendiker $M$, Veprintsev D, et al. Inhibiting HIV-1 integrase by shifting its oligomerization equilibrium. Proc Natl Acad Sci USA 2007; 104:8316-21.

50. Kramer-Hammerle S, Ceccherini-Silberstein F, Bickel C, Wolff $\mathrm{H}$, Vincendeau M, Werner T, et al Identification of a novel Rev-interacting cellular protein. BMC Cell Biol 2005; 6:20.

51. Zhang J, Scadden DT, Crumpacker CS. Primitive hematopoietic cells resist HIV-1 infection via p21. J Clin Invest 2007; 117:473-81.

52. Yamamoto N, Tanaka C, Wu Y, Chang MO, Inagaki $\mathrm{Y}$, Saito Y, et al. Analysis of human immunodeficiency virus type 1 integration by using a specific, sensitive and quantitative assay based on real-time polymerase chain reaction. Virus Genes 2006; 32:105-13.

53. Field FJ, Born E, Murthy S, Mathur SN. Polyunsaturated fatty acids decrease the expression of sterol regulatory element-binding protein- 1 in $\mathrm{CaCo}-2$ cells: effect on fatty acid synthesis and triacylglycerol transport. Biochem J 2002; 368:855-64.

54. Casabianca A, Gori C, Orlandi C, Forbici F, Federico Perno C, Magnani M. Fast and sensitive quantitative detection of HIV DNA in whole blood leucocytes by SYBR green I real-time PCR assay. Mol Cell Probes 2007; 21:368-78.

55. Levin A, Kutznetova L, Kahana R, Rubinstein-Guini M, Stram Y. Highly effective inhibition of Akabane virus replication by siRNA genes. Virus Res 2006; 120:121-7. 